\title{
Rapports entre La Police et le Judiciaire dans la Procédure d'Éxécution de la Peine*.
}

Paulo José da Costa Jr.

Professeur de Droit Pénal dans les Universités de S. Paulo et Roma.

1.0. Observation préliminaire: L'éclosion du phénomène criminel déclenche un processus de rapports complexes entre l'organe policier et l'autorité judiciaire. Ce processus comporte trois phases distinctes, la dernière ayant été inexplicablement omise dans l'ensemble des thèmes proposés. En effet, les deux phases initiales de cette série de rapports on été envisagées. D'abord la phase de l'investigation, dans laquelle sont récueillies des informations fondamentales sur lesquelles, virtuellement, s'établira la manifestation de la prétension punitive. Ensuite, la phase du procés, dans laquelle, formellement, la prétension punitive est exteriorisée, passée au crible du contradictoire et finalement accueillie ou repoussée par la décision judiciaire. Il apparaît ainsi que la phase tertiaire, celie de l'éxécution de la peine, phase dans laquelle le "jus puniendi" est materialisé, a été passée sous silence. Or, il s'agit là d'une lacune qu'il faut combler, puisque dans le cadre de la réalité moderne c'est justement dans cette phase tertiaire que les rapports entre la police et l'organe judiciaire se présentent comme les plus délicats et les plus inquiétants.

* Relação apresentada e unanimemente aprovada no IV Simpósio Internacional de Criminologia Comparada, realizado pela Universidade de Montreal, em abril de 1972. 
1.1. Véritablement, "dans le contexte des sociétés les plus industrialisées, les policier est appelé à jouer un rôle de plus en plus complexe et, à certains égards, polyvalent". Il faudrait donc examiner, du point de vue de la procédure d'éxécution de la peine, si cette complexité et cette polyvalence qui caractérisent les fonctions policières dans la société moderne sont des attributs salutaires, à envisager avec indifférence, ou, au contraire, s'ils suggèrent l'émergence d'un phénomène inquiétant d'hypertrophie fonctionnelle.

Nous essayerons d'analyser, d'abord d'un point de vue général, la complexité et la polyvalence des fonctions sociales dans la société moderne, pour voir dans quelle mesure les fonctions, qu'elles soient remplies indistinctement par des personnes publiques ou par des personnes privées, entrent en concurrence avec d'autres à l'intérieur de l'organisme social dès qu'elles deviennent polyvalentes. Nous verrons ensuite que, virtuellement, la polyvalence de certaines fonctions implique dans l'hypertrophie de ces fonctions et dans l'atrophie correspondante et nécéssaire de la fonction concurrente, pour vérifier que, plus spécialement en ce qui concerne la procédure d'éxécution de la peine, la polyvalence observée des fonctions de police se traduit dans la pratique par un ammoindrissement ou même par l'annulation du contrôle jurisdictionnel qui doit s'exercer sur la phase éxécutive du jus puniendi.

2.2. L'affirmation selon laquelle les rapports sociaux dans le monde contemporain se caractérisent par leur bouleversante complexité est un véritable truisme. Non seulement le savent ceux qui par leur responsabilité scientifique ou définition professionnelle sont concernés par l' analyse du phénomène social, mais l'a aperçu aussi d'une façon ou d'outre l'homme ordinaire, par intuition et par experience. Même sans être un observateur méthodique, celui-ci se sent également étourdi par la complexité des situations 
qui l'entourent, situations dont il participe en sa vie grégaire.

Nul n'ignore plus de nos jours que cette complexité augmente à mesure que le processus d'industrialisation s'oriente vers le stade post-capitaliste de production, stade qui aurait déjà été atteint par certains secteurs de la communauté internationale, à en croire certains auteurs.

Le monde où nous vivons s'est transformé en villageglobal, oeuvre de l'inépuisable criativité de la technologie des comunications, en supermarché planétaire, où les incitations universelles à la consommation substituent ce que l'on appelle les intérets de l'Etat et où la dynamique imposée par les groupements industriels multinationaux devient plus importante que les stratégies politiques encore conçues en fonction du système des frontières. Il $\mathrm{y}$ a néanmoins un bouleversant paradoxe dans ce monde où l'homogénéisation s'accentue de plus en plus, où l'éxotisme des anciennes habitudes disparaît devant l'uniformité des usages imposées par les déluges publicitaires qui nivelent les hommes et uniformisent leurs nécéssités. Le monde se réduit à un immense bourg, mais les rapports sociaux n'obéissent plus au même ritme ordonné, précis et harmonieux qui caractérisait les anciens rapports villageois.

Voilà ce qui étourdit: les hommes vivent dans le même village, répondent aux mêmes stimulus, sont victimes des mêmes anxiétés, ont des nécésités égales et partagent les mêmes ambitions. Mais, contrairement à ce qui se produisait dans les villages pré-capitalistes et même dans les sociétés émérgentes des premières étapes de la révolution industrielle, l'homme ne discerne plus avec la même assurance le rôle qui lui revient dans la dynamique du village, ni ne réussit pas à définir avec précision sa véritable place dans l'ensemble de la structure.

Curieusement nous sommes revenus au village, mais celui-ci ne correspond plus, définitivement, que cela conforte 
ou que cela déprime, à l'image que nous en faisions, peutêtre par atavisme, peut-être en fonction d'une formation culturelle anachronique. Nous sommes revenus au village, mais pas au conformisme de son engrenage hiérarchisé, où chacun avait sa fonction et son rêve, diversement reservés et suggerés par la paillasse où par le baldaquin.

L'homme du village-global, à la différence de ce qui faisaient monotonement ses aïeux il y a peu de temps encore, ne repète plus les expériences accumulées par sa famille au long des siècles. Il ne doit plus forcément être ce que son père et son grand-père on été, et souvent il n'est plus aujourd'hui ce qu'il avait été jusqu'hier. Le commis voyageur devient du jour au lendemain le puissant patron d'une chaîne de grands magasins. La célérité vertigineuse qui caractérise la circulation de la monnaie transforme le millionnaire d'hier dans l'assalarié d'aujourd'hui. L'actionnaire majoritaire constate, vaguement surpris, que son pouvoir de propriétaire n'est que fictif, puisqu'en fait c'est le technocrate qui tire les ficelles dans son entreprise. Dans le village-global il n'y a plus de châteaux impérissables ni de destins éternels, il n'y a plus de fonctions privilégiées, intouchables, ni de centres de pouvoir absolu.

Des phénomènes déjá suffisament analysés et classés, comme par exemple celui de la mobilité sociale toujours croissante, mettent en cause les vieux concepts de stratification sociale et engendrent des genres de rapports extrêmement complexes, et en même temps changeants. Les habitants du village-global, toujours psychologiquement influencés par les conditionnements dérivés de l'infra-structure périmée, n'ont pu s'y conformer et s'y adapter sans accuser l'impact de traumatismes douloureux.

L'habitant du village-global comprend ou au moins s'aperçoit que son activité, sa participation, son existence sociale enfin, ne sont plus circonscrits à un domaine net et définitif de contribution, que l'on pense au plan horizontal 
ou au plan vertical. Il a la conscience, ou au moins la sensation qu'aucune fonction spécifique ne lui est pas inéxorablement réservée dès sa naissance jusqu'à sa mort par un déterminisme social qui ne conserve plus la totalité des hommes soumise aux caprices du démiurge. Le roi sera roi, le vassal sera vassal. Il constate, effrayé parfois, que dans le village-global il n'y a plus une place unique qui lui soit réservée, puisque si formellement ses fonctions sont encore statiques et définies, en réalité ses responsabilités se multiplient et deviennent de plus en plus complexes, sa mission polyvalente. Il ne doit pas obéir ou commander, il obéit et il commande en même temps. Il se soumet à un chronograme et à un organograme de travail certes, mais la rapidité avec laquelle changent les techniques et les nécéssités l'oblige à trouver constamment des solutions nouvelles, en même temps qu'elle lui offre la possibilité d'échapper à la tyranie des programmes et d'imposer sa propre volonté. C'est pour cela que les dénommés hommes d'initiative assument une telle importance. Dès qu'un homme peut prendre des initiatives, il n'a plus une fonction délimitée, il prend en charge toutes les fonctions qui l'entourent.

La conscience du fait que $s a$ responsabilité est marquée par la fluidité et que $s a$ fonction renferme potentiellement ou en fait toutes les fonctions est une source d'angoisse pour l'habitant du village-global. Il est pris par la nostalgie de l'époque où, définis son office et son devoir, l'homme envisageait son destin avec ennui ou même avec dégoût parfois, mais toujours avec une tranquilisante sensation de sécurité.

Il découvrait son futur dès le moment où il reconnaissait é immutabilité de son propre destin. Il vivait continuellement d'accord avec sa propre identité, persuadé que cette identité échappait aux contingences et aux surprises. Il faut remarquer que cette sensation de sécurite ne concernait que les valeurs matérielles, quotidiennes, mais aussi e surtout une 
philosophie de vie. Comme l'habitant du village-national (idée opposée et corrélative à celle de village-global) connaissait ce que l'on nommait sa place et corrélativement son devoir, il était exempté d'avance de toute responsabilité concernant les vicissitudes qui ne lui aparaîssaient pas comme strictement et rigoureusement dérivées de sa fonction et de son devoir spécifiques, étant ainsi déchargé d'une quelcounque fraction de culpabilité. Il était compromis avec l'ordre social d'une façon abstraite, mais aliéné par rapport à ses dernières conséquences. Le cordonnier ne devait s'occuper quedes semelles, il n'avait rien à voir avec les insuccès militaires du roi.

L'homme du village-global, par contre, est persuadé du fait qu'il est engagé dans toutes les responsabilités, même dans celles qui régardées d'un point de vue extérieur et immédiat ne semblent pas concerner ses fonctions et ses devoirs. Comme corolaire de cette polyvalence fonctionnelle en société qu'il pressent, il ne peut pas éloigner de son esprit les virtuels sentiments de culpabilité. Il ne peut plus comme dans le passé avoir recours aux subterfuges consentis, selon lesquels sa responsabilité ne dépasserait pas les limites étroites de son office, les murs de sa maison, l'enceinte de son groupe ou même de sa classe. Cela est vrai pour les personnes physiques comme pour les personnes juridiques. Un syndicat ouvrier en grève, dans les sociétés hautement développées, transporte toute la vie nationale à l'intérieur de son propre domaine, tandis que le dirigeant syndical acquiert, pendant un certain laps de temps, un pouvoir équivalent à celui $d \mathfrak{u}$ chef de l'Etat. Corrélativement, le patron d'un “holding" tentaculaire n'ignore pas que ses décisions ont des répercussions profondes, ni que les sysmographes économiques enregistreront l'impact de ses décisions dans les recoins les plus éloignés du village-global. $\mathrm{Ni}$ le dirigeant syndical, ni l'entrepreneur monopoliste ne peuvent ôter leurs éventuels sentiments de culpabilité sous pretexte 
que leur responsabilité est stricte. Ils savent qu'elle ne l'est pas.

2.1. Dans une société où les fonctions sont d'une telle manière poìyvalentes, répandues, concurrentes, il n'y a plus comme autrefois de soupapes de sûreté pour les sentiments de culpabilité. L'inexistence de soupapes de sûreté providentielles suscite, il est notoire, l'éclosion de mécanismes de défense qui déterminent l'élimination des sentiments de culpabilité par voie de l'engourdissement des scrupules. Comme il ne sait pas exactement quelle est sa fonction dans l'organisme social, et comme il s'aperçoit que cette fonction est ménacée par l'identique perplexité de son voisin, l'homme du village-global se permet d'avancer de plus en plus, ne fuyant ni l'usurpation des fonctions, ni l'accumulation du pouvoir. Comme il doit subir l'angoisse de supporter toutes les responsabilités, que lui Soit an moins consentie la satisfaction de réunir toutes les fonctions, sans se préoccuper avec les problèmes d'autrui. Et le village-global ne se soustrait pas ainsi à l'empire de cette règle si inhumaine qu'inéxorable: la survie du plus fort, l'extinction du plus faible.

Il est vraissemblable que ce sentiment de responsabilité globale, d'une part, et la répulsion naturelle à la loi du plus fort, d'autre part, soient à l'origine de l'angoisse qui a favorisé, dans ce fin de siècle, l'éclosion des mouvements de masses poussées par l'envie desesperée d'un retour à la vie communale primitive, aux formes artisanales de travail, aux modes d'existence dans lesquels l'homme connaît precisément $s a$ fonction et son devoir, donc, son destin. Les mouvements "hippie" seraient peut-être l'exemple le plus suggestif de ce phénomène, de cette volonté de retour aux modèles sociaux où l'individu ne se sent pas pressé, par des sollicitations multiples et par des convocations pressantes, à assumer des responsabilités polyvalentes, dont la prise en 
charge implique certainement dans l'étouffement des responsabilités d'autrui.

2.2. Il est évident que si nous avons poussé ces considérations à un niveau paroxystique c'est simplement pour faire ressortir l'intérêt de l'analyse et pour faire remarquer la vérité qui, nous en sommes persuadés, en découle. Si nous avons laissé transparaître une vision apocalyptique des rapports sociaux dans le village-global, cela se doit seulement, il faut le souligner, au désir d'enregistrer dramatiquement notre conviction: Dans le cadre de la société moderne, la compléxité des fonctions engendre inéluctablement des conflits qui sont d'autant plus inquiétants qu'il nous est permis de prévoir que certaines fonctions qui sont chères à notre esprit libéral et à notre formation humaniste risquent d'être absorbées par d'autres, dans lesquelles nous ne pouvons pas reconnaître avec égale facilité la même noblesse.

3.0. Tout ce qui a été dit à propos de l'individu dans le village-global peut être et doit être généralisé tout court, de façon que l'on puisse reconnaître dans les fonctions des personnes publiques la même polyvalence que l'on a prétée aux activités sociales des personnes privées.

Les objectifs de ce travail, tout comme la courte haleine de son auteur, ne comportent pas une analyse profonde des fonctions de l'Etat dans le monde contemporain, ou, pour garder le langage que nous avons employé dès le début, des fonctions de l'Etat dans le village-global.

Il faut néanmoins signaler, quoique de manière sommaire, que le concept d'Etat est en train de subir une procédure de révision, parfois radicale, qui correspond à la nécéssité scientifique d'adapter le concept traditionnel à une réalité visiblement metamorphosée. Au sommet de cette procédure de révision sont agitées des formulations vraiment bouleversantes pour les esprits de formation orthodoxe (devrait-on dire libérale?): ainsi celle qui pro- 
clame la faillite dans la pratique de la théorie de la séparation des pouvoirs, telle que l'a conçue Montesquieu, comme celle qui sous pretexte que le pouvoir ne provient pas du peuple, mais de l'aptitude personnelle pour son exercice, propose le transfert de tous les pouvoirs d'Etat au mandarinat contemporain, à la technocratie, ou encore celle qui, en renversant les notions consacrées de souverainété, préconise la reconnaissance de frontières supra-nationales, démarquées au gré des intérêts idéologiques et commerciaux.

3.1. Il est certain que dans le village-global, comme les fonctions de l'individu, les fonctions classiques de l'Etat sont devenues difuses, multiples, polyvalentes. Ceux qui ont pris l'habitude de les identifier par leur apparence continuent seuls à voir une démarcation nette de ces fonctions, circonscrites par les lignes institutionnelles qui théoriquement fixent Ieur sphère de compétence. Celui qui dépasse les apparences, avec esprit critique et lucidité scientifique, peut néanmoins constater, malgré son instinctive résistance, que les fonctions étatiques débordent souvent le cadre institutionnel, de telle façon que certaines de ces fonctions s'accroissent, tandìs que d'autres, corrélativement, se réduisent. Le phénomène de l'hypertrophie du Pouvoir éxécutif est aujourd'hui evident en tous les recoins du village-global. C'est justement parce que nous vivons dans un village-global que les phénomènes n'ont pas un domaine limité, national, d'incidence.

I'attachement romantique à l'idée que la structure du pouvoir et les sphères de compétence des fonctions étatiques on été définitivement modelées par la doctrine de ce siècle et se trouvent cristalisées dans les textes constitutionnels en vigueur est inutile, irrationnel, anti-scientifique. La réalité pratique montre que cette structure ne présente pas l'articulation harmonieuse des fonctions qu'avait conçue la dogmatique juridico-politique née de l'espoir caractéristique des périodes d'après-guerre. 
Il apparaît comme une évidence que les fonctions, les sphères de compétence, les formes d'exercice du pouvoir sont tellement entrelacées dans l'Etat $d u$ Village-global que bientôt il sera difficile, sinon impossible, de distinguer où commencent les unes et où finissent les autres, malgré les rigides limites constitutionnelles.

3.2. Le juriste attentif à ces transformations sociales doit idéaliser et mettre à l'épreuve des formules nouvelles de contrôle mutuel dans le domaine des rapports entre différentes fonctions de l'organisme étatique, allant jusqu'à proposer le sacrifice d'une de ces fonctions, au nom de la préservation de celle qui se présente dans la pratique comme la moins apte à se défendre et idéalement comme la plus souhaitable. Cette philosophie nouvelle de comportement, qui implique pour le juriste en la renonce à l'étrange et ingénue croyance dans les solutions de niveau purement constitutionnel, recommande des mesures pratiques et extrêmes: lorsqu'il estime qu'une fonction considérée comme noble est ménacée d'absortion, le juriste doit conseiller des mesures d'amputation ou, au moins, des mesures capables de contenir la menaçante hypertrophie de la fonction considérée comme la moins souhaitable.

En partant de la prémisse selon laquelle la compléxité des rapports sociaux dans le monde contemporain détermine l'éclosion des phénomènes de phagocytose fonctionnelle, entre organes qui théoriquement ne devraient pas être concurrents, mais qui en réalité le sont, le juriste doit manifester sa préférence, applaudir ou condamner l'absorption observée, de manière nette et objective, sans faire naivement confiance à la capacité des solutions consacrées par les textes légaux de discipliner, à elles seules, la concurrence des attributions, en arrêtant la croissance démesurée d'une fonction au détriment de l'autre. Il doit, finalement, abandonner l'illusion que la physiologie sociale finit toujours pour trouver 
des solutions dictées par ses propres sègles et recommander avec courage des propositions chirurgicales.

4.0. Dans la perspective que nous sommes en train de développer, ce qui nous inquiète particulièrement c'est le fait que la fonction policière absorbe de plus en plus la responsabilité effective de la procédure d'éxécution de la peine. Le fait que la fonction juridictionnelle est de plus en plus absente dans cette phase tertiaire de la persecutio criminis nous préoccupe intensement, une fois que toutes les raisons conduisent à la soluction opposée: il faut réserver au judiciaire la part la plus grande de responsabilité pour atteindre le but thérapeutique de la peine.

4.1. La procédure d'éxécution de la peine est universellement laissé au soin de la fonction policière. D'autre part, la règle consacrée, réitérée par le $\mathrm{X}^{\mathrm{e}}$ Congrès International de Droit Pénal de Rome, préscrit que cette procédure doit se dérouler sous contrôle juridictionnel.

En théorie, donc, les rapports entre la fonction policière et la fonction juridictionnelle dans la procédure d'éxécution de la peine suggèrent une relation harmonieusement articulée, conformément à la solution consacrée par les textes légaux. Lorsque la décision condamnatoire devient définitive, la fonction policière se charge de mettre en éxécution les dispositions de la sentence. La fonction judiciaire doit veiller pour que la thérapeutique appliquée par l'organe policier s'accomplisse strictement selon les limitations de la prétension punitive à satisfaire in casu et encore pour que cette thérapeutique s'oriente sans écarts vers la réalisation des objectifs rédempteurs qui inspirent l'application de la peine.

Des raisons d'ordre doctrinnaire et d'ordre pragmatique conduisent néanmoins à ne pas croire à l'éfficacité de cette formule.

On doit considérer d'abord que l'éxécution de la peine, qui réprésente l'instauration d'un nouveau et vrai procès, d'une nouvelle et authentique relation juridico-procéssuelle, 
où, si on veut, le prolongement du procès qui a abouti à l'adoption de l'alternative condamnatoire, ne peut pas échapper au contrôle direct de la fonction juridictionnelle. Qu'elle soit une nouvelle relation juridico-processuelle ou le prolongement éxécutoire du procès (les objectifs de cet exposé ne comportent pas la discussion de la nature juridique de l'institut), l'éxécution de la peine doit se dérouler sous la présidence du même organe qui a dirigé le développement de la procédure déclaratoire de la responsabilité pénale. Les mêmes motifs, doctrinaires et pratiques, qui soumettent la procédure déclaratoire de la responsabilité pénale à la compétence de la fonction juridictionnelle se présentent lorsqu'il s'agit de la procédure de sublimation de la prétention punitive. Pourquoi donc ne conférer qu'une simple fonction de contrôle à l'organe juridictionnel dans la procédure d'éxécution de la peine, alors que dans la procédure antérieure il avait aussi une fonction éxécutive (ordinatoire)?

Il nous semble, de plus, que la dichotomie fonctionnelle qui préside à l'éxécution de la peine - - disons-le froidement - est en contradiction avec la conception moderne concernant le but de la peine. Il nous semble en effet refléter un souvenir du temps où la peine n'avait qu'un caractère rétributif. Il ne semblait pas alors impropre que l'organe juridictionnel, après avoir décreté la sanction prévue par la loi, eût donné sa mission pour terminée et eût lavé les mains, comme Pilate, transférant à d'autres l'éxécution de la volonté de la loi. La solution était très convenable: si on avait pour but, avec la peine, d'affliger le condamné, il était plus raisonnable que des hommes non professionnellement imprégnés des idéaux de Justice se chargeassent de cette tâche, pour épargner aux hommes de la Justice le malaise d'éxécuter personnellement des pratiques affligeantes.

Aujourd'hui néanmois cette dichotomie fonctionnelle ne se justifie plus, à moins que l'on veuille que l'humanisation de la peine dont on parle tellement, l'évolution pro- 
clamée de la peine à la catégorie de mesure de récupération, ne soient un exemple de plus de l'âbime que sépare l'idéal de la réalité, dont se complait tellement l'hypocrisie humaine. L'organe juridictionnel ne peut pas, en silence et avec résignation, voir trahis les propos rédempteurs qui ont inspiré l'application de la sanction légale. Il doit, au contraire, prendre en charge la responsabilité de l'éxécution de la peine, effectivement et intégralement, pénétré des mêmes intentions humanitaires qui ont dicté son application. Si la peine n'est pas une méthode de rétribution, mais de purification, il est convenable que le Magistrat qui l'a déclarée nécéssaire en contrôle l'éxécution, pour l'orienter dans le sens de la réalisation de ses intentions cathartiques. C'est peut-être parce que les juges de la Sainte Inquisition se fondaient en des considérations de cet ordre qu'ils dirigeaient personnellement la procédure éxécutoire des peines qu'ils appliquaient.

4.2. On pourra éventuellement objecter qu'en vérité l'organe juridictionnel ne renonce pas à cette responsabilité, puisqu'il exerce un contrôle sur la procédure d'éxécution de la peine, et que ce contrôle est le quantum satis pour prévénir la possibilité de déformations pratiques. De cette façon la question de l'intrégrité des objectifs idéaux de la peine est un sujet qui ne concerne qu'un plus grand ou un plus petit dégré de la vigilance éxercée par le judiciaire.

En réponse, il faut affirmer tout de suite que nous insistons en voir le problème du point de vue de la concurrence entre fonctions, avec toutes les implications et les risques dont nous avons parlé dans les considérations introductoires. Nous croyons que la procédure d'éxécution de la peine signale de manière croissante la prédominance de la fonction policière, c'est-à-dire, enregistre l'atrophìe galopante de la fonction juridictionnelle dans ce domaine. Nous estimons que le simple contrôle n'est pas un moyen propre à renverser cette dangereuse tendance. 
Il faut encore souligner que la réalité démontre l'inneficacité d'un tel contrôle. En effet, il est possible d'observer que le contrôle juridictionnel de l'éxécution de la peine est souvent pro forma. Il ne consiste qu'en des visites sporadiques du Magistrat à l'établissement pénitentiaire ou en des rencontres non moins éparses entre le Magistrat et celui qui a obtenu la liberté conditionnelle. Or, que l'on n'ait pas la velléité d'affirmer que ce contrôle $\dot{a}$ distance, divorcé de la réalité des prisons, du au jour le jour de l'éxécution de la peine, ne fondé souvent qu'en des fichiers et documents des archives et réalisé presque toujours à cause d'obscures curiosités statistiques, soit suffisant pour assurer la réalisation des buts thérapeutiques de la peine.

Ce contrôle à distance n'est pas capable d'empêcher que le traitement de récupération du condamné subisse les distortions que l'on connaît. Et il ne l'empêche pas, en effet, puisque les indices de reincidence deviennent de plus en pIus aigus, ce qui démontre que le condamné, fréquemment soumis à une reformatio in pejus si authentique que déplorable, est rendu à la société en des conditions de détérioration morale et physique plus graves et plus accentuées que celles presentées au début de l'éxécution de la peine. En réalité, ce contrôle à distance ne s'est pas révélé capable d'assurer l'ntègrité du droit primaire et fondamental du condamné, le droit d'être rendu à la société au moins en des conditions equivalentes à celles où il se trouvait lorsqu'il est entré dans l'établissement d'internement. En d'autres termes, s'il n'est pas possible d'opérer une reformatio in melius, il doit être assuré au condamné qu'il ne subisse pas une reformatio in pejus, devant être rendu à la société au moins dans le statu quo ante (restitutio in integrum).

4.3. Nous sommes pérsuadés que, dans le domaine de l'éxécution de la peine, la fonction juridictionnelle s'atrophiera gradduellement, jusqu'a ce qu'elle soit totalement 
absorbée par la fonction policière, autant que persiste la vélleité de considérer que sa responsabilité se limite à la simple opération de contrôle.

De plus, contrôler l'éxécution de la peine, honnêtement, c'est vivre au jour le jour l'éxécution de la peine. Or, la fonction policière est la seule qui participe de cette vie de chaque jour. L'organe policier est le seul personnage que le condamné rencontre regulièrement, jour par jour, heure par heure, minute par minute, tandis que se déroule le drame de la procédure éxécutoire pénale. C'est exactement pour cela que la fonction policière est en train de prendre en charge toutes les responsabilités concernant l'éxécution de la peine, pour en donner sa propre orientation. Elle institue les normes d'administration pénitentiaire, influe sur les conceptions architectoniques des maisons d'internement, arrivant jusqu'à concevoir les préceptes de droit positif en matière pénitentiaire. Les réprésentants de la fonction policière sont aujourd'hui, sans aucune doute, les technocrates les plus versès en matière de science pénitentiaire. L'organe policier concentre tout le pouvoir d'initiative, ef quand le pouvoir d'initiative est donné à un organe, - l'idée a déjà été exprimée ci-dessus - il n'a plus une fonction délimitée, il englobe toutes les fonctions limitrophes. Dans les cadres de la fonction juridictionnelle il a peu de connaîsseurs de la technique d'éxécution pratique de la peine, et ceux là le sont presque toujours par dilettantisme.

Ainsi, le dénommé contrôle juridictionnel de l'éxécution de la peine devient de plus en plus un contrôle restreint, rélégué à une situation platonique, limité à la fiscalisation de certaines règles minimales. La fonction juridictionnelle est réleguée à une situation bureaucratique subalterne, limitée à l'exercice d'un certain nombre de rites anodins et de formalités qui n'influent pas fondamentallement sur les résultats pratiques de la peine: contrôle des délais, notes diverses etc..

Il faut remarquer que toutes les fois que la fonction juridictionnelle s'impatiente, s'inquiète avec cette position 
subalterne et platonique et essaye de prendre effectivement en charge: ses prérogatives de contrôle pour vaincre la sensation d'atrophie progressive, des phénomènes de friction apparaîssent, des heurts se produisent et alors émergent nettement les symptomes de la dégénerescence des limites des sphères de compétence. Il apparaît alors que certaines fonctions ont tendance à prédominer dans un système de concurrence qui, s'il est salutaire du point de vue de l'initiative privée, est indiscutablement nocif du point de vue de la mission étatique, puisque il est à la source de la dévaluation de la notion globale d'autorité.

En reprenant le fil de notre exposé, il faut insister sur le point qu'il est inutile de nier cette évidence: l'organe, l'autorité, ou plus directement l'éxécuteur de la fonction, chargé des ordres, arbitrages immédiats et de prendre toutes dispositions pendant la procédure d'éxécution de la peine sera le détenteur des vrais pouvoirs de contrôle sur la procédure éxécutoire. Le fonctionnaire chargé de la surveillance du condamné est celui qui en réalité soulage ou éxaspère le châtiment. Il est le seul responsable de l'éventuelle récuperation du condamné à qui il a dévoué un intérêt fraternel, ou de la dégénerescence absolue de celui à qui il a réservé l'implacabilité du bourreau.

La responsabilité concernant l'éxécution de la peine est néanmoins une matière qui doit être proposée et conçue ratione officii; la responsabilité de la procédure éxécutoire pénale appartient à la fonction juridictionnelle, et son efficacité ne peut rester dans la contingence du caractère plus ou moins compatissant ou plus ou moins truculent de l'agent de la fonction policière.

4.4. Quelle doit être l'attitude du juriste qui reconnaît de manière honnête et réaliste que le dénommé contrôle juridictionnel de l'éxécution de la peine est quelque chose de purement platonique et inneficace en ce qui concerne le but espéré d'assurer la réhabilitation morale du con- 
damné? Doivent-ils continuer à croire naivement que les solutions législatives disciplinant les rapports entre les fonctions de l'organisme étatique soient suffisantes pour empêcher que la fonction juridictionnelle soit absorbée par la fonction policière?

Doivent-ils non moins naivement multiplier les textes réglementaires, dans le but de renforcer les limites de la sphère de compétence, estimant ainsi que la pléthore des proclamations lègales est un remède capable de soumettre les fonctions concurrentes à un système artificiel d'équilibre?

$\mathrm{Ne}$ devraient-ils, imbus d'un critère plus réaliste, conseiller l'adoption de dispositions plus effectives, de mesures plus conrageuses, énergiques, positives?

Si la fonction juridictionnelles est en train de perdre de l'importance en ce qui concerne l'éxécution de la peine, tandis que la fonction policière s'accroît et se consolide, il faut avant tout que l'on fasse une option concernant la survie de l'une ou de l'autre, parce que toute attitude moins radicale sera dépourvue de conséquences. Ne pas trancher, c'est-à-dire être indifférent devant le phénomène de l'absorption ou essayer de concilier les fonctions, c'est le même que décreter l'éloignement complet, à moyenne échéance, de la fonction juridictionnelle en ce qui concerne la phase de l'éxécution de la peine. Et cela répresente, que l'on le veuille ou non, une option en faveur de la prépondérance de la fonction policière.

Ainsi, celui qui désire réaliser une option en faveur de la fonction juridictionnelle devra sans hésiter s'engager dans le combat pour que la fonction concurrente soit exclue de l'éxécution de la peine. Il devra lutter pour que la fonction juridictionnelle, qui sans doute contient aussi en elle des attributions de caractère administratif, soit hissée à une situation de pouvoir réel. Les Magistrats, où encore les réprésentants du Ministère Public, doivent prendre en charge la responsabilité de la diréction des établissements péniten- 
tiaires, de la sélection de personnel effectivement qualifié pour les fonctions auxiliaires, de la prévention de la réincidence. cette dernière responsabilité pouvant être exercée directement ou par des organes délégués, dans le sens de maintenir une vigilance effective sur ce aux qui obtiennet la liberté conditionnelle et de maintenir et administrer des maisons d'accueil et des institutions du même genre.

Nous sommes convaincus que la fonction juridictionnelle doit être protégée contre l'absorbante hypertrophie de la fonction policière, cette dernière ne nous paraissant pas plus doué que la fonction juridictionnelle pour prendre en charge la responsabilité d'assurer l'éfficacité thérapeutique de la peine. On l'a remarqué ailleurs, "l'agent de police, par la propre nature de ses activités ordinaires, a toujours tendance à voir dans le delinquent, même dans celui qui a obtenu la libération conditionnelle comme récompense pour sa bonne conduite, un ennemi de la société." L'homme qui est en prison ou qui en est sorti "doit rencontrer non pas une vigilance pleine de peur et de soupçon, mais une simpathique attention, prête à tout moment à lui donner du courage dans le maintien de son propos de méner une vie honnête. La police ne peut pas le faire, parce qu'il ne s'agit pas de sa tâche habituelle et parce que cette attitude est contraire à sa psychologie professionnelle, orienté dans le sens de guetter le delinquent, et, pourrions-nous ajouter, parce que la police est motivée par une notion souvent très litérale et très agréssive de ce que doit être sa mission de "guerre au crime", notion qui lui inculque une position aguérrie, position qui est certainement propre à la consécution de sa mission préventive, mais incompatible avec la mission thérapeutique.

En conclusion:

Dans le village-global, lorsqu'on s'apperçoit qu'une fonction présente une tendance à l'absorption d'une autre, dans le cadre de ses rapports de plus en plus complexes, entrelacés, confus, et lorsqu'on veut à tout prix préserver la fonction menacée, parce que celle ci apparaît comme la 
plus noble ou comme la plus compatible avec la nature de la mission dont elles disputent l'éxécution, il est inutile d'essayer de rétablir une harmonie théorique: la fonction la plus proche de la réalité, celle qui exerce un pouvoir réel sur l'objet, finira pour éclipser l'autre fonction. Il est plus pratique et plus conséquent d'empêcher qu'elles s'affrontent dans la même sphère diaphane de compétence; il faut renforcer la fonction choisie, en même temps que vider complètemente la fonction moins désirable. Les paliatifs son innéficaces, la solution chirurgicale s'impose.

Si ces propositions sont valables, son extrapolation pour le domaine de l'éxécution de la peine mène à la conclusion que la fonction juridictionnelle sera inéluctablement absorbée par la fonction policière, à moins qu'une option radicale entre l'une et l'autre soit imposée. Nous croyons que cette option doit favoriser la fonction juridictionnelle, la seule effectivement préparée par sa psychologie professionnelle, dont les attributs sont la serenité, l' impartialité et l'équilibre, pour prende les dispositions qui s'imposent pour que la peine aboutisse à ses buts idéaux. Nous sommes presque tentés de proposer l'adoption d'un mot d'ordre grandiloquent: en matière d'éxécution de la peine, - tout le pouvoir au Magistrat! 